\title{
Geokodierte Meldedaten als Basis bedarfsgerechter Planungen in ländlichen Gemeinden und Regionen
}

\author{
Markus Schaffert ${ }^{1}$. Volker Höcht ${ }^{2}$ \\ Eingegangen: 30. August 2017 / Angenommen: 31. August 2018 / Online publiziert: 14. September 2018 \\ ๑) Springer-Verlag GmbH Deutschland, ein Teil von Springer Nature 2018
}

\section{Zusammenfassung}

Einwohnermeldedaten können einen wichtigen Beitrag zur Unterstützung der raumbezogenen Planung in ländlichen Gemeinden leisten. Das zeigen mehrere Studien, die sich in den vergangenen Jahren mit demographischen Veränderungsprozessen und Versorgungsbedarfen in Deutschlands ländlichem Raum auseinandergesetzt haben. Gemeinsam ist diesen, meist einzelfallbezogenen Untersuchungen, die Geokodierung kommunaler Melderegister und ihre analytische Verarbeitung mittels Geographischen Informationssystemen. Auf diese Weise lassen sich Bevölkerungsstrukturen und ihre Veränderungen auch unterhalb der Gemeindeebene auflösen und in Analysen nutzen, während Bevölkerungsdaten der überkommunalen statistischen Stellen dies in der Regel nicht vermögen. Dieser Beitrag greift Erkenntnisse aus diesen Studien auf. Er verbindet sie zu einem Argumentationsstrang, der Mehrwerte des Ansatzes sowohl für die kommunale als auch die regionale Ebene aufzeigt und anhand von Beispielen aus Bayern und Sachsen-Anhalt entwickelt wird. Neben allgemeinen demographischen Aspekten werden dabei auch „Erreichbarkeiten“ und „Immobilien“ - als mit demographischen Prozessen in Zusammenhang stehende Themenfelder - behandelt. Die Darstellung der Mehrwerte, die zu einer stärkeren Nutzung von Melderegistern in Wissenschaft und planerischer Praxis motivieren möchte, wird ergänzt um eine Diskussion über die Grenzen des verfolgten Ansatzes im Hinblick auf zukünftige Bevölkerungsentwicklungen. Durch das Aufzeigen sowohl von Mehrwerten als auch von Grenzen der Nutzung geokodierter kommunaler Bevölkerungsdaten möchte der Beitrag zudem die Umsetzung von Digitalisierungsstrategien in der Planung ländlicher Räume unterstützen.

Schlüsselwörter Melderegister · Geographische Informationssysteme · Inner- und überkommunale Analysen · Ländliches Demographiemanagement

Dr. Markus Schaffert

schaffert@gih.uni-hannover.de

$\bowtie$ Dr. Volker Höcht

volker.hoecht@hs-anhalt.de

1 Geodätisches Institut, Leibniz Universität Hannover, Nienburger Straße 1, 30167 Hannover, Deutschland

2 Institut für Geoinformation und Vermessung, Hochschule Anhalt, Bauhausstraße 8, 06846 Dessau, Deutschland 


\title{
Geocoded Data from Population Registers as a Source for Needs-Based Planning in Rural Municipalities and Regions
}

\begin{abstract}
Population registers can make an important contribution to spatially related planning in rural municipalities. This has been illustrated in a number of recent studies that have examined processes of demographic change and service requirements in Germany's rural areas. These investigations are usually case-study based and share an approach involving the geo-coding of municipal population registers and their analysis using Geographical Information Systems. This reveals and allows the analysis of population structures and changes thereto, also for units below the municipal level, as is rarely possible when using population data held by supra-municipal statistical authorities. This paper draws on findings from these investigations. It combines them to form a line of argument that demonstrates the value of the approach for both the municipal and the regional levels, using illustrative examples from Bavaria and Sachsen-Anhalt. In addition to general demographic aspects, the focus is on "accessibility" and "real estate" as topics that are associated with demographic processes. Discussion of the value of this approach is intended to motivate the increased use of population registers both in academic research and in planning practice. Attention is also drawn to the limits of the approach with a view to future population trends. By highlighting both the value and the limits of the use of geo-coded municipal population data the paper also aims to support the implementation of digitalisation strategies in the planning of rural areas.
\end{abstract}

Keywords Population register - Geographic information systems $\cdot$ Local and regional analyses $\cdot$ Rural demography management

\section{Einleitung}

Die statistischen Ämter der Länder und des Bundes stellen eine Vielzahl von Daten über Bevölkerungsstruktur und -veränderung in ihrem Zuständigkeitsgebiet bereit. Allerdings bilden diese Daten nicht diejenige räumliche Ebene $a b$, die für angemessene Entscheidungen in Deutschlands Kommunen mitunter erforderlich ist. So sind die Datenprodukte der statistischen Ämter in der Regel auf die gesamte Gemeinde oder auf höhere administrative Einheiten aggregiert. ${ }^{1}$ Gemeinden müssen sich dagegen mit Problemlagen auseinandersetzen, die regelmäßig eine räumlich genauere Betrachtung erfordern. Typische Fragen in diesem Zusammenhang lauten: „Wo innerhalb einer Gemeinde werden Einfamilienhäuser der Nachkriegsjahrzehnte nur noch von wenigen hochbetagten Bürgern bewohnt?“, „In welchen Ortsteilen ist die Zahl leerstehender Wohngebäude besonders hoch?“ oder „Wie lassen sich Standorte von Versorgungseinrichtungen und deren Erreichbarkeit optimieren, um der demographischen Situation im Ort gerecht zu werden?"

Mit demographischen Daten, die Informationen nur für die Gesamtgemeinde bieten, lassen sich Fragen dieser Art nicht passgenau beantworten. Allerdings existiert mit dem

\footnotetext{
${ }^{1}$ Daten des Zensus 2011, die auf www.zensus2011.de öffentlich bereitgestellt werden, sind nicht auf administrative Einheiten, sondern auf 100x100m-Raster aggregiert. Sie bieten insofern zwar innerörtliche Informationen, allerdings keine Adressgenauigkeit. Da sie zudem in der Regel nicht aktuell vorliegen - die UN empfiehlt einen Aktualisierungszeitraum von zehn Jahren (Scholz/Kreyenfeld 2016: 3) - werden sie hier nicht näher betrachtet.
}

Melderegister eine durch die Gemeinden geführte Datenquelle, die sich für kleinräumige demographische Untersuchungen eignet. So enthalten Einwohnermeldedaten zentrale demographische Informationen der Einwohner einer Kommune (z. B. Geburtsdatum, Geschlecht und Staatsangehörigkeit) sowie einwohnerbezogene Veränderungen (z. B. Zuzugs- und Wegzugsdatum). Zudem werden diese Informationen gemeinsam mit der Adresse der Einwohner geführt, was räumliche Analysen innerhalb des Gemeindegebietes ermöglicht. Wird die Adresse in Realweltkoordinaten transformiert (,geokodiert"; vgl. Waldner/Simonet/ Machguth et al. 2005), können Meldedaten über den gemeinsamen Raumbezug zudem mit weiteren Geobasis- und Geofachdaten kombiniert werden. Kleinräumige Bevölkerungsdaten werden so zu einer Basisinformation, die unter anderem mit Daten zur Siedlungsentwicklung, zum Flächenverbrauch oder zu Versorgungsbedarfen in Zusammenhang gebracht werden kann.

Allerdings hat das Melderegister in seinem gesetzlich definierten Beschreibungsumfang - und in der Folge im Funktionsumfang der Software, mit denen das Melderegister in deutschen Kommunen geführt wird - das Meldewesen und nicht die kommunale Planung im Fokus. Seine Aufbereitung zu Planungszwecken benötigt deshalb in nicht unwesentlichem Maße Personal- oder Finanzressourcen sowie Expertenwissen, das von technischer Befähigung bis hin zu datenschutzrechtlicher Bewandtnis reichen sollte. Die systematische Verwendung von geokodierten Meldedaten für weiterführende Fragestellungen stellt gerade in ländlichen Gemeinden, die, anders als größere Städte, häufig über keine Statistikabteilungen mit geschultem Personal und einem 
gesetzlich legitimierten Rahmen verfügen, deshalb nicht die Regel dar (vgl. Haußmann 2012).

In den vergangenen Jahren sind mehrere Veröffentlichungen erschienen, die die Bedeutung von Einwohnermeldedaten zur Unterstützung der raumbezogenen Planung in ländlichen Gemeinden belegen und dabei auf die Anwendung von Geographischen Informationssystemen (GIS) ${ }^{2}$ setzen. So untersuchen Schaffert (2008), Kötter/Schollän (2010), Koppers/Baumann (2011), Höcht/ Weichert/Pickelmann (2012), Benndorf/Bergfeld/Schaffert et al. (2013) sowie Köhler (2014) auf diese Weise den Einfluss von demographischen Prozessen auf kommunale Ver- und Entsorgungsbedarfe und deren Infrastrukturen. Allgemeine demographische Analysen als kleinräumige Informationsbasis für Dorferneuerungsmaßnahmen erarbeiten Schaffert/Müller/Benndorf (2011) und Lang/Zettler (2012), während Planinsek (2011) auf den Generationenwechsel in alternden Einfamilienhausgebieten blickt. Rößler (2011), Koppers/Bergfeld/Höcht et al. (2014) sowie Köhler/Schaffert (2015) wiederum untersuchen Wanderungsbewegungen auf der Grundlage von Einwohnermeldedaten und interessieren sich dabei auch für interkommunale Zusammenhänge. $^{3}$

Die zuvor genannten, vornehmlich analytisch orientierten Arbeiten werden durch Ansätze der technischen Werkzeugentwicklung ergänzt. So entwickelt Huber (2015) eine zentrale Adressdatenverwaltung für kommunale Planungsund Verwaltungsaufgaben auf der Grundlage des Melderegisters. Darüber hinaus wurden intra- oder internetbasierte Softwarelösungen zur Unterstützung der Regionalplanung (Vilser 2014; Kirschke 2015) und für das gemeindliche Immobilienmanagement (Schaffert 2010; Höcht/Kirschke 2017) vorgestellt. Bei Letzteren handelt es sich um GISKomponenten, deren Funktionalität - z. B. durch vereinfachte Nutzerführung und übersichtliche Programmoberflächen - auf die Zielgruppe „kommunale Verwaltung“ optimiert wurde. Diese Softwarelösungen, die unter Verwendung von Meldedaten arbeiten, wurden für Dörfer und Kleinstädte in ländlichen Räumen konzipiert, wenngleich sie prinzipiell unabhängig von ländlichen oder städtischen Fragestellungen sind.

\footnotetext{
${ }^{2}$ „Ein Geographisches Informationssystem ist ein rechnergestütztes System, das aus Hardware, Software, Daten und den Anwendungen besteht. Mit ihm können raumbezogene Daten digital erfasst und redigiert, gespeichert und reorganisiert, modelliert und analysiert sowie alphanumerisch und graphisch präsentiert werden." (http://www. geoinformatik.uni-rostock.de/einzel.asp?ID=793 (27.06.2018)).

3 Methodisch vergleichbare Ansätze mit überwiegend städtisch geprägten Untersuchungsgebieten (z. B. Föbker 2008; Rudolph-Cleff 2012; Benneter 2013) oder ohne konkreten Bezug zu ländlichen Räumen (z. B. Sturm/Meyer 2009) werden an dieser Stelle nicht vertiefend betrachtet.
}

Vorschläge zur systematischen Integration von melderegister- und GIS-basierten Bevölkerungsanalysen oder Werkzeugen in strategische Planungsansätze unterbreiten Schaffert (2011a) für die Ergänzung der Szenarioplanung in schrumpfenden Dörfern, Farrokhikhiavi/Dietrich/ Vallée (2013) für eine Regionalstrategie Daseinsvorsorge sowie Schaffert (2011b) und Neufeld/Beyrich/Burkhardt et al. (2015) für das Leerstandsmanagement ländlicher Wohngebäude. Höcht (2016a, 2016b) bezieht räumliche Melderegisteranalysen als Basis in die Konzeption eines strategischen Demographie-Managements für Kleinstädte in ländlichen Schrumpfungsregionen ein.

Anders als die genannten Untersuchungen blickt der vorliegende Beitrag nicht auf bestimmte ländliche Gemeinden oder auf thematische Einzelaspekte kommunalstatistischer Analysen. Ebenso wenig geht es um die Entwicklung eines spezifischen Werkzeugs zur Unterstützung dieser Analysen. Anliegen ist vielmehr, einen Überblick über Einsatzmöglichkeiten geokodierter Melderegister für den planerischen Umgang mit demographischen Prozessen in ländlichen Gemeinden und Regionen zu bieten. Dazu werden diese Einsatzmöglichkeiten für die kommunale Ebene (Kapitel 2; am Beispiel von Flossenbürg) und für Regionen (Kapitel 3) beschrieben. Wesentliche Aspekte werden mittels eines Argumentationsstranges erklärt, der anhand von Fallstudien aus Bayern und Sachsen-Anhalt entwickelt wird und neben allgemeinen demographischen Aspekten auf die Themenfelder „Immobilien“ (Kapitel 3.1; am Beispiel Wunsiedel) und „Erreichbarkeit“ (Kapitel 3.2) referiert. Er dient dazu, Mehrwerte darzustellen, die sich aus der Verarbeitung von Einwohnermeldedaten mittels Geographischer Informationssysteme und deren Bereitstellung in Geodateninfrastrukturen ergeben (Kapitel 3.3; am Beispiel von Gardelegen und Möckern). Ergänzend kommen Grenzen des verfolgten Ansatzes in Bezug auf kleinräumige Bevölkerungsvorausberechnungen zur Sprache (Kapitel 4; am Beispiel der Marktgemeinde Teisnach). ${ }^{4}$ Ein Fazit mit Ausblick über die Verwendung geokodierter Melderegisterdaten steht in Kapitel 5.

\footnotetext{
${ }^{4}$ Grenzen aufgrund von datenschutzrechtlichen Gegebenheiten werden in den nachfolgenden Ausführungen ebenfalls benannt. Eine ausführliche Diskussion dieses Themas unterbleibt jedoch. In Zeiten der Digitalisierung und der Evolution rechtlicher Rahmenbedingungen - so wurde die Datenschutzgrundverordnung (DSGVO) am 25. Mai 2018 in der gesamten Europäischen Union wirksam und unterstützt einheitliche Regelungen zum Schutz von personenbezogenen Daten - zeigt dieser Beitrag nüchtern Vorteile der planerischen Nutzung von Meldedaten auf. Diese Vorteile bieten in der Debatte um künftige rechtliche Regelungen, Verstöße und Entwicklungen - vgl. z. B. o. V. (2018) zum Vorschlag des Städte- und Gemeindebundes über den erweiterten Verkauf von anonymisierten Kommunaldaten Orientierung.
} 


\section{Demographische Analysen innerhalb des Gemeindegebietes: die kommunale Perspektive}

In kommunalen Melderegistern werden, wie zuvor genannt, wesentliche demographische Eigenschaften jedes in einer Gemeinde gemeldeten Bürgers erfasst. Infolgedessen bieten sie sich als zentrale Informationsquelle für ein kommunales Demographiemanagement an (vgl. Höcht 2016a). Da die demographischen Eigenschaften gemeinsam mit der jeweiligen Meldeadresse geführt werden, lassen sie sich in einem Geographischen Informationssystem verarbeiten und auf diese Weise in räumlichen Zusammenhängen darstellen.

Abbildung 1 zeigt exemplarisch das Durchschnittsalter der Einwohner der Gemeinde Flossenbürg in Nordbayern (rund 1.700 Einwohner). ${ }^{5}$ Hinsichtlich des Verwendungszwecks - im konkreten Fall handelt es sich um eine Informationsgrundlage für Workshops mit Bürgern - wurde eine Dichtekarte erstellt, die eine leicht verständliche Kommunikation der Information verspricht und durch die Darstellung von Umfeld- anstatt Adressinformationen den Rückschluss auf einzelne Personen und Adressen verhindert. Das Durchschnittsalter wurde aus den im Melderegister geführten Geburtsdaten errechnet. Entsprechend lassen sich alle im Melderegister geführten Attribute unmittelbar kartographisch abbilden oder als Basis für mehr oder weniger komplexe Berechnungen nutzen. ${ }^{6}$

Ein kommunales Demographiemanagement sollte neben kleinräumigen demographischen Informationen auch solche zur Immobiliensituation einer Gemeinde umfassen (vgl. Höcht 2016a). So bieten Informationen über Gebäude planerisch und städtebaulich relevante Kenngrößen und ergänzen allgemeine demographische Informationen sinnvoll (vgl. Bürkner/Berger/Luchmann et al. 2007). Zudem beeinflusst etwa die Altersstruktur der Bewohner den Zeitpunkt von Besitzerübergängen bei Einfamilienhäusern, die in ländlichen Räumen verbreitet sind (Zakrzewski/ Berndgen-Kaiser/Fox-Kämper et al. 2014). Das Melderegister bietet nicht nur Informationen zu demographischen Eigenschaften der Hausbewohner. Es lassen sich auch Aspekte mit unmittelbarem Bezug zu den Gebäuden ableiten. So können unbewohnte Gebäude identifiziert werden, indem (gemäß Melderegister) bewohnte Adressen der Gesamtzahl aller Adressen einer Gemeinde gegenübergestellt werden. Dies lässt sich in einem Geographischen Informationssystem durch Verknüpfen von Meldedaten mit den

\footnotetext{
5 Das Rastergitter, das zur Darstellung der Ergebnisse dient, beträgt im konkreten Fall 5 Meter.

${ }^{6}$ Einer komplexeren Berechnung bedarf beispielsweise das Billeter$\mathrm{Maß}$ zur Beschreibung der Altersstruktur, das durch Schaffert/Müller/ Benndorf (2011) und Lang/Zettler (2012) in einem Geographischen Informationssystem umgesetzt wurde.
}

Hauskoordinaten ${ }^{7}$ einer Gemeinde über die gemeinsame Adressangabe realisieren (vgl. Spehl 2011). Die nichtverknüpfte Restmenge enthält die unbewohnten Gebäude einer Gemeinde zusammen mit denjenigen Adressen, an denen keine Wohnnutzung vorgesehen ist. Durch anschließendes Verknüpfen der Restmenge mit Daten zu gewerblich bzw. öffentlich genutzten Immobilien kann die Aussagekraft der Ergebnisse weiter verbessert werden (vgl. Schaffert 2011a). Dieses Verfahren lässt sich automatisieren und ist mittlerweile in der Praxis angekommen. So fand es als tragende Säule des sogenannten Baulücken- und Leerstandskatasters Eingang in ein Produkt des niedersächsischen Landesamts für Geoinformation und Landesvermessung, das den Kommunen des Landes als Dienstleistung angeboten wird (Kleinwächter 2014).

Diese Herangehensweise führt zu einer räumlichen $\mathrm{Ag}$ gregation der ,rohen ' Melderegister-Daten: Während im Melderegister einzelne Personen als zentrales Identifikationsobjekt fungieren, werden nun Sacheigenschaften der in einem Gebäude wohnhaften Personen räumlich auf die Adresse zusammengefasst. Ergebnisse solcher Analysen lassen sich in einem Geographischen Informationssystem flexibel auch auf höhere Raumeinheiten - im Gemeindegebiet z. B. Baugebiete, Ortsteile oder Straßenräume beziehen. ${ }^{8}$ Exemplarisch zeigt Abbildung 2 für die Gemeinde Flossenbürg die Anzahl von Gebäuden, in denen wenige und zugleich alte Bewohner leben (nachfolgend als Remanenzgebäude ${ }^{9}$ bezeichnet) - aggregiert auf den Ortskern sowie auf Siedlungserweiterungsgebiete.

Die Verknüpfung mit weiteren Geodaten erlaubt es, zusätzliche Informationen über Lageparameter abzuleiten, die die Attraktivität einer Immobilie beeinflussen und im $\mathrm{Zu}$ sammenspiel letztlich auch die Leerstandsgefährdung von Wohngebäuden erhöhen können (vgl. Schaffert 2011b). Als Beispiele lassen sich Nähe zu Durchfahrtsstraßen, Bebauungsdichten oder die Erreichbarkeit von Einrichtungen der Grundversorgung anführen (vgl. Abbildung 3). Solche Analysen können zu wesentlichen Teilen anhand von amtlichen Geobasisdaten, die in Kommunen bereits vorliegen

\footnotetext{
7 Dies ist ein amtliches Datenprodukt der Vermessungsverwaltungen der Bundesländer, das Koordinaten aller Adressen einer Gemeinde umfasst.

${ }^{8}$ Dies gilt jedenfalls, insofern flächenhafte (polygonale) Geodaten, die diese höheren Raumstrukturen repräsentieren, vorliegen oder im Geographischen Informationssystem (z. B. als Rastergitter oder als sogenannte Pufferzonen) neu geschaffen werden.

9 Altersremanenz bezeichnet das Verbleiben älterer Bewohner im zuvor gemeinsam bewohnten Haus nach dem Auszug der Kinder (Raab 2006: 555). Die Kinder verlassen das Elternhaus (oft ein Einfamilienhaus in Eigentum), um einen eigenen Haushalt zu gründen und gegebenenfalls selbst Wohneigentum zu erwerben. Das Elternhaus spielt für die Entscheidung der Kinder bezüglich Wohneigentum oft keine Rolle. Denn es ist regelmäßig nicht ,frei ', wenn sich die Kinder in dem Alter befinden, in dem Menschen Wohneigentum schaffen.
} 
Abbildung 1 Beispiel für nutzergerecht aufbereitete kleinräumige demographische Informationen $^{10}$

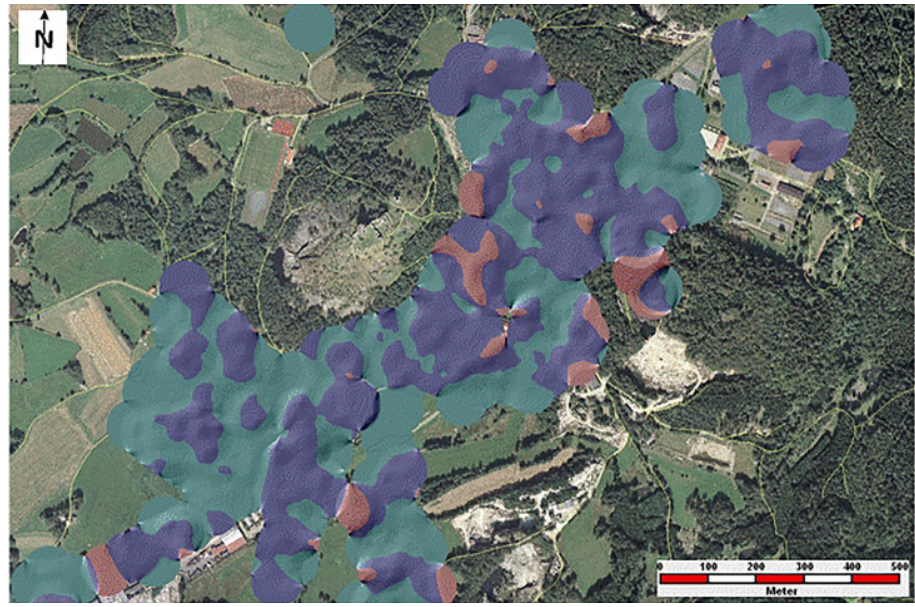

Durchschnittsalter

in Flossenbürg

Darstellungsart:

Schummerung

Basis der Berechnung:

$\varnothing$-liches Alter je Adresse

über 65 Jahre

45 bis 65 Jahre

unter 45 Jahre

Abbildung 2 Anteil von Remanenzgebäuden am Gebäudebestand Quelle: Schaffert (2011a: 110)

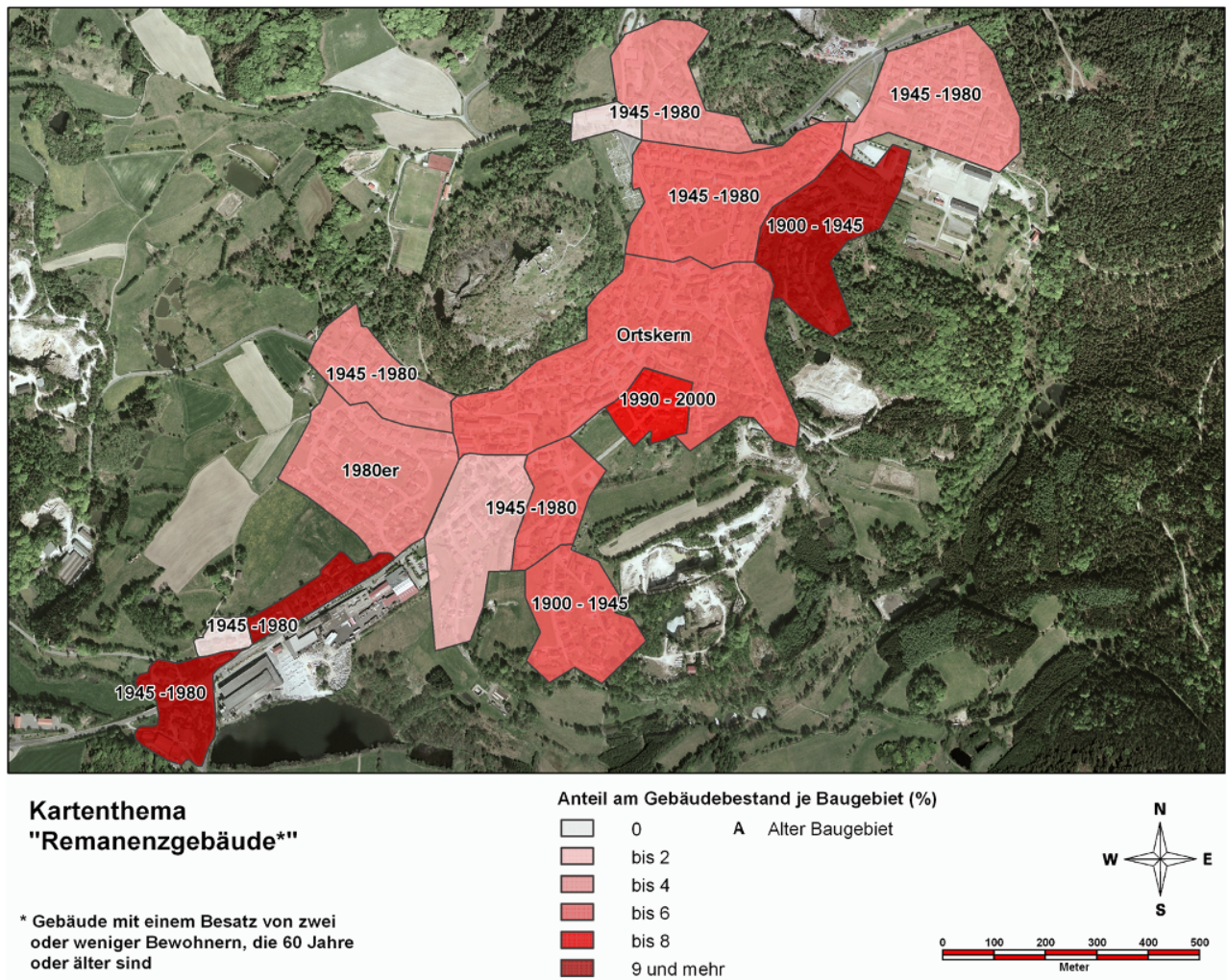

oder - bei Berücksichtigung ihrer räumlich sowie zeitlich abweichenden Datenqualitäten - gegebenenfalls auch mit „Crowd-Sourced"-Geodaten ${ }^{11}$ durchgeführt werden (vgl. Schaffert 2011a).

Der Vergleich der Abbildungen 1 und 2 lässt erkennen, dass das südwestliche Erweiterungsgebiet, das in den

\footnotetext{
${ }^{10}$ Das Orthofoto im Hintergrund wurde vom Landesamt für Digitalisierung, Breitband und Vermessung Bayern zur Verfügung gestellt.

11 „Crowd-Sourced“-Geodaten (auch ,Volunteered Geographic Information") sind von Freiwilligen erhobene raumbezogene Daten. Das wohl bekannteste Projekt ist OpenStreetMap.
}

Jahren von 1945 bis 1980 in der Gemeinde Flossenbürg bebaut wurde, neben einer relativ hohen Zahl von Remanenzgebäuden, auch ungünstige fußläufige ${ }^{12}$ Erreichbarkeiten von Versorgungseinrichtungen des täglichen Bedarfs aufweist. Dieser Umstand vermag das Interesse potenzieller

\footnotetext{
12 Als Grenze akzeptabler Erreichbarkeiten zu Fuß wurden hier 500 Meter angenommen (vgl. http://www.sozialplanung-senioren.de/dasinstrument/indikatoren-themenfeld-3-wohnen-und-lebensfuehrung/ 33-infrastruktur/index.html (29.06.2018)). Die Distanz ist im GIS flexibel wählbar und auf spezifische Fragestellungen und Ausgangssituationen anderer Kommunen anpassbar. Die verwendeten Straßendaten stammen von www.OpenSteetMap.com.
} 
Abbildung 3 Erreichbarkeit von Einrichtungen der Grundversorgung Quelle: Schaffert (2011a: 114)

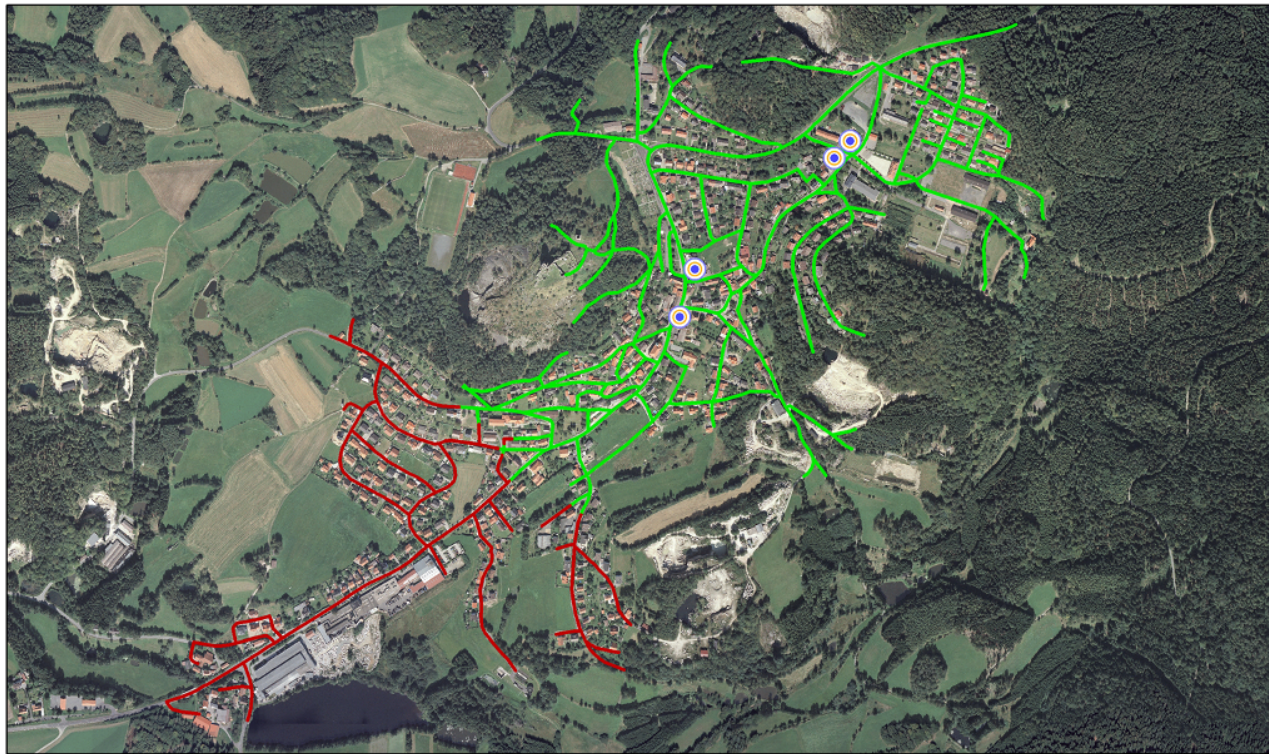

$$
\begin{aligned}
& \text { Entfernung zu "Lebensmittel-Versorgern" in Flossenbürg } \\
& \begin{array}{l}
\text { Grundversorgung Lebensmittel (Standorte: Bäcker, Metzger, Supermarkt) } \\
\text { über } 500 \text { Meter } \\
\text { bis zu } 500 \text { Meter }
\end{array}
\end{aligned}
$$
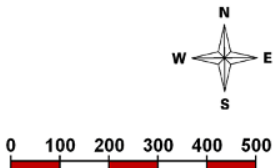
Meter

Abbildung 4 Entfernung zwischen Infrastruktureinrichtung und Adressen als ,Träger demographischer Information“ Quelle: Benndorf/Bergfeld/Schaffert et al. (2013: 17)

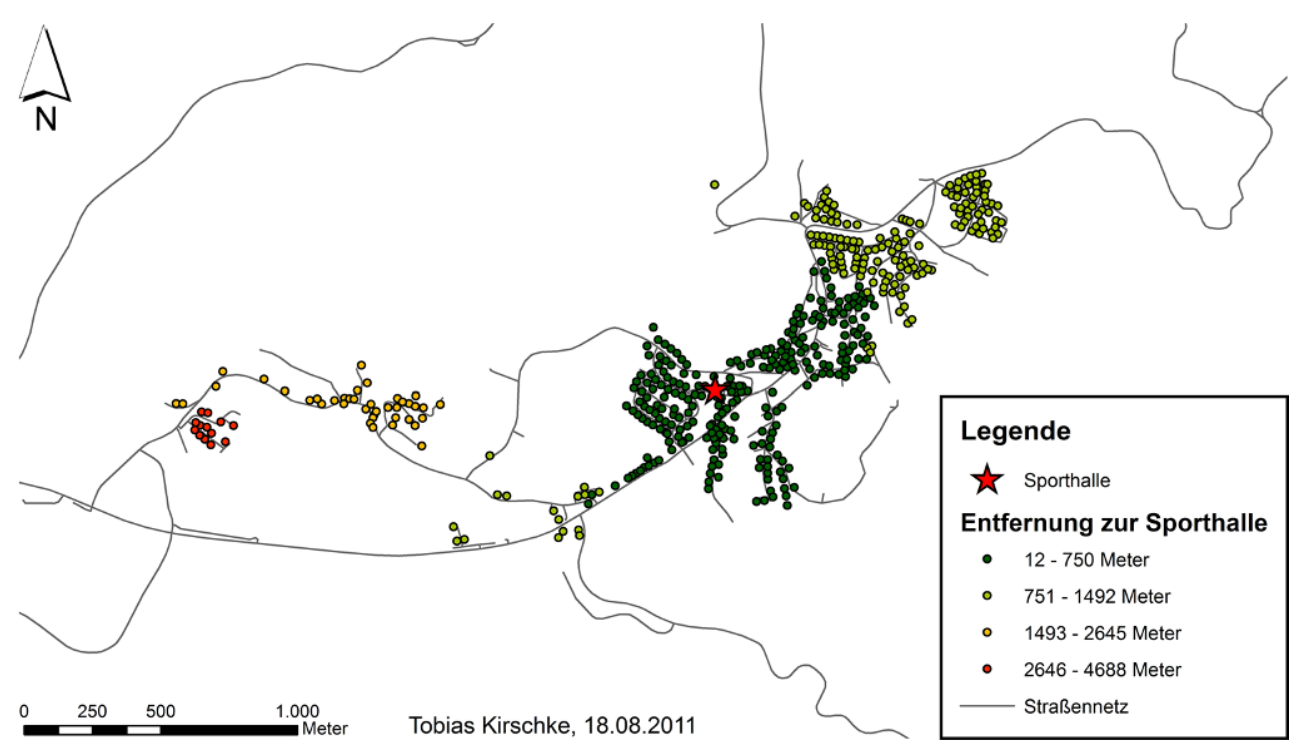

Käufer an dort gelegenen Immobilien nach ihrem lebenszyklusbedingten Leerfallen gegebenenfalls verringern (vgl. Zakrzewski/Berndgen-Kaiser/Fox-Kämper et al. 2014). Erreichbarkeiten von Bäcker, Metzger oder Supermarkt sind jedoch nicht nur Parameter, die Gebäudeattraktivitäten beeinflussen. Sie sind auch für die Versorgung einer alternden Bevölkerung von zentraler Bedeutung (Farrokhikhiavi/ Dietrich/Vallée 2013).

Karrais (2014: 56) konstatiert, dass Erreichbarkeitsberechnungen seit den 1970er-Jahren fester Bestandteil der
Forschung sind. Allerdings „beziehen sie häufig nur die Distanz oder Fahrzeit als Kriterium in die Berechnung der Erreichbarkeit mit ein" (Karrais 2014: 56). Dieser Befund gilt auch für die Anwendung von Erreichbarkeitsanalysen zur Untersuchung von Versorgungslagen, die verbreitet ohne Integration von Bevölkerungsdaten in die Berechnung erfolgt. Die Aussagekraft von Erreichbarkeitsanalysen lässt sich durch die Verwendung von Bevölkerungsdaten jedoch erhöhen. So erlauben Meldedaten Erreichbarkeitsberechnungen, die Personen in bestimmten Lebensphasen als Aus- 
gangspunkt haben und sich auf von diesen benötigten Infrastruktureinrichtungen, wie Kindergärten, Gesundheitszentren, Schulen oder Sporteinrichtungen, beziehen. Dahinter verbirgt sich mehr als das gemeinsame Betrachten und Vergleichen von - gegebenenfalls für einen Workshop kartographisch aufbereiteten - Einzelanalysen. Das Prinzip verdeutlicht Abbildung 4: Ausgehend von Standorten von Infrastruktureinrichtungen (hier: eine Sporthalle) werden Erreichbarkeiten (hier: die Entfernung zur Sporthalle in Metern) berechnet und zugleich auf adressgenaue demographische Informationen bezogen. Dies führt zu einem analytischen Mehrwert, da das Frequentieren von Infrastruktureinrichtungen vom Alter bzw. von Lebensphasen potenzieller Nutzer abhängt. Die Abbildung zeigt aus Gründen des Datenschutzes die Entfernung der Sporthalle zu allen Adressen der Beispielkommune. Für die interne Verwendung können mit dem gleichen Prinzip Erreichbarkeiten auch ausschließlich zu Adressen mit Bewohnern in relevanten Lebensphasen - sei es für den Jugendbasketball, die Seniorengymnastik oder für weitere Zwecke - berechnet werden (vgl. Koppers/Baumann 2011).

Liegen keine Meldedaten vor wird mitunter der Ansatz verfolgt, kleinräumige demographische Daten durch sogenannte Disaggregation (vgl. Burgdorf 2010) zu generieren (Schwarze 2008; Neumeier 2017). Dabei werden räumlich zusammengefasste Bevölkerungsdaten auf kleinere Einheiten verteilt, wobei dies oft anhand der Bebauungsstruktur erfolgt. Ausgehend von Bevölkerungsdaten für die gesamte Gemeinde würden einem Ortsteil mit vielen Mehrfamilienhäusern mehr Bewohner zugewiesen als einem Ortsteil mit überwiegend lockerer Einfamilienhausbebauung. Diese Herangehensweise erscheint für kleinräumige Untersuchungen jedoch nicht unproblematisch. So dürfte sich von der Bebauung oftmals nicht auf die Bevölkerungsstruktur der Bewohner einzelner Gebäude schließen lassen. Ist in einem Einfamilienhaus der 1960er- oder 1970er-Jahre der Generationenwechsel bereits vollzogen und lebt dort wieder eine junge Familie mit Kindern oder sind es noch ein oder zwei Personen der alternden Erstgeneration? Anzahl und Alter der Bewohner von besagtem Einfamilienhaus lassen sich aus Meldedaten ermitteln (vgl. Planinsek 2011). Sie können seriöserweise aber nicht aus gesamtgemeindlichen Statistikdaten disaggregiert werden.

\section{Demographische Analysen oberhalb der Aggregationsebene "Gemeinde": die regionale Perspektive}

Demographische Prozesse machen nicht an Gemeindegrenzen halt. Deshalb ist neben der Untersuchung kleinräumig-innerörtlicher Gegebenheiten die über- und zwischenkommunale Situation von Bedeutung. Dies gilt nicht zu- letzt auch für die zuvor unter Fokussierung auf kommunale Zusammenhänge beschriebenen Konsequenzen von demographischen Prozessen hinsichtlich Immobilien und Erreichbarkeiten. Darüber hinaus fordern begrenzte finanzielle Handlungsspielräume der Kommunen eine verstärkte interkommunale Zusammenarbeit.

\subsection{Immobilien und Wanderungsmotive}

Möchte eine Kommune ein Immobilienmanagement unter Beachtung von demographischen Gesichtspunkten etablieren, so ist neben dem Verständnis innerkommunaler Immobiliensituationen und Einwohnerstrukturen eine zusätzliche überkommunale Perspektive unerlässlich. So dürfte beispielsweise eine Person, eine Familie bzw. ein Haushalt, die bzw. der aus Arbeitsplatzgründen in eine bestimmte ländliche Region zieht, ihre Immobilienentscheidung aufgrund von Immobilienverfügbarkeiten und Preisen, der Nähe zur neuen Arbeitsstelle, familiären Motiven und sonstigen Lagefaktoren auf der Mikro- und Makroebene treffen (vgl. Bauer/Holz-Rau/Scheiner 2005). Die jeweilige Gemeinde - ihr Name, ihre Geschichte, ihre Verwaltungsstruktur und weitere kommunale Eigenheiten - erscheinen aus dieser Sicht als eher zweitrangig für die Standortentscheidung zugunsten von Wohnraum. Diese Annahme wurde in der nordbayerischen Kleinstadt Wunsiedel (rund 10.000 Einwohner) in städtischem Auftrag überprüft, um eine solide Entscheidungsgrundlage für das kommunale Immobilienmanagement zu schaffen (vgl. weiterführend Koppers/Bergfeld/ Höcht et al. 2014; Höcht 2016a: 102 ff.). Da das Melderegister sowohl die frühere als auch die aktuelle Meldeadresse führt, erlaubt es die Analyse von $\mathrm{Zu}$-, Weg- und innerkommunalen Umzügen und stellt deshalb auch für Wanderungsuntersuchungen eine geeignete Informationsbasis dar.

Obwohl die Zahl der sozialversicherungspflichtig beschäftigten Arbeitnehmer in der Kleinstadt in den Jahren zuvor stetig gestiegen war, herrschte bei kommunalen Akteuren die Ansicht vor, der migrationsbedingte Verlust an Bevölkerung sei insbesondere auf arbeitsmarktbezogene Faktoren zurückzuführen. Regionen mit einem attraktiven Arbeitsmarkt, wie Nürnberg oder München, wurden entsprechend als beliebte Ziele der Wegziehenden vermutet. Die Analyse der Zieldestinationen im Geographischen Informationssystem zeigte jedoch, dass die Wanderungsbewegungen weg aus der Untersuchungskommune überwiegend innerhalb der Region - und dort vornehmlich im Nahbereich - stattgefunden hatten (vgl. Abbildung 5). Zudem ließen sich ähnliche Arbeitsmarktstrukturen in den Zieldestinationen und der Untersuchungsgemeinde nachweisen, weshalb die vermeintlich ausschlaggebende Bedeutung arbeitsmarktbezogener Kriterien für die Wohnortwahl in Frage gestellt wurde (Höcht 2016a: 102ff.). 
Abbildung 5 Wanderungsziele der aus Wunsiedel Weggezogenen Quelle: Koppers/Bergfeld/ Höcht et al. $(2014: 6)^{13}$

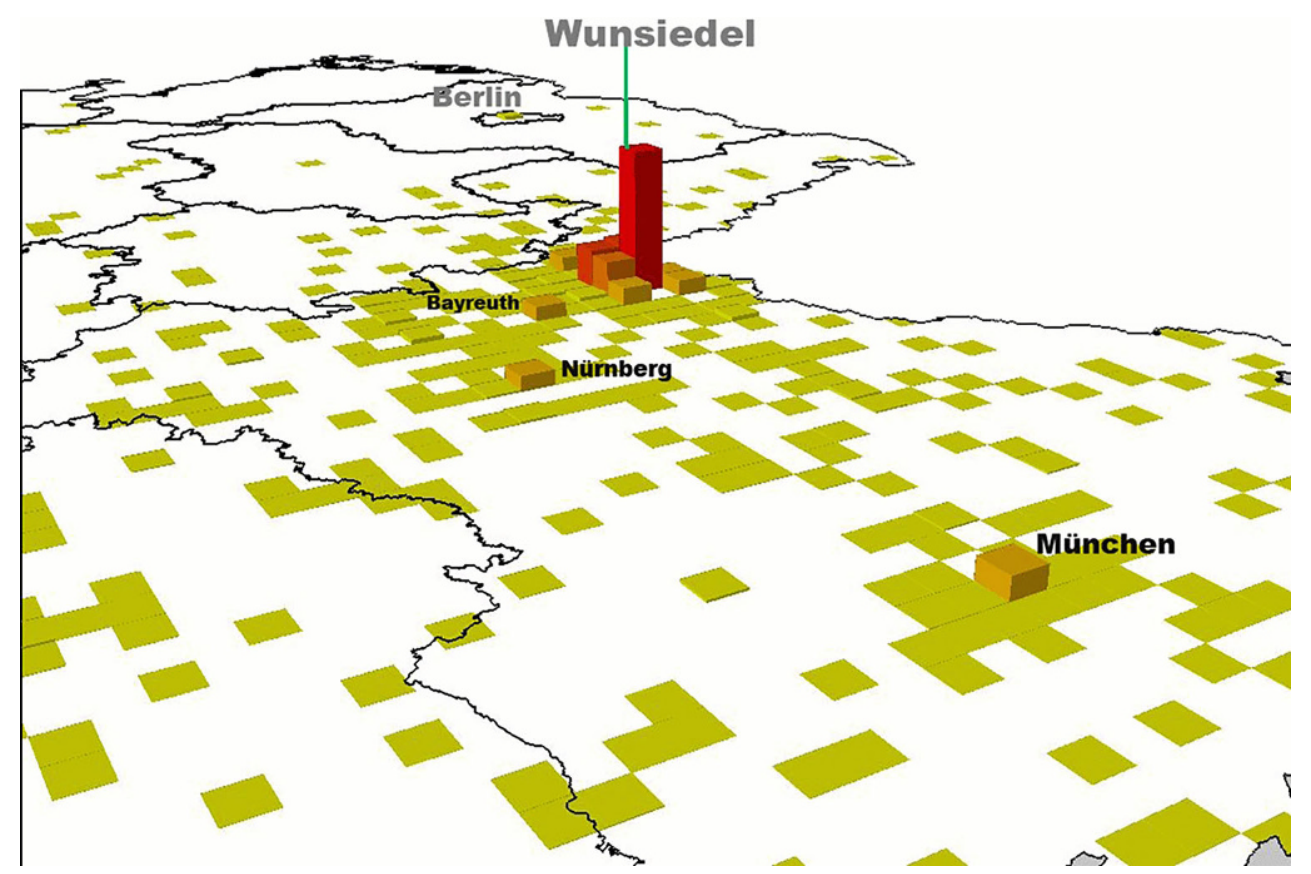

Neben dem Arbeitsmarkt gelten insbesondere Faktoren des Familienzyklus (z. B. Geburten oder der Tod eines Partners) und auf Immobilien bezogene Aspekte als bedeutsam für Wanderungen innerhalb von Regionen (Föbker 2008: 43 ff.; Wehrhahn/Sandner Le Gall 2011: 110). In Ergänzung der meldedatenbasierten Analyse räumlicher Wanderungsmuster wurde deshalb in einem zweiten Schritt eine leitfadengestützte Telefonbefragung von zirka 1.000 $\mathrm{Zu}$ - und Weggezogenen (der Jahre 2001 bis 2010) im Alter zwischen 18 und 80 Jahren durchgeführt. ${ }^{14}$ Neben Wanderungsmotiven wurden dabei auch Stärken und Schwächen der betrachteten Kleinstadt Wunsiedel abgefragt, um daraus Rückschlüsse für die Ausrichtung der Stadtentwicklungsplanung ziehen zu können.

Im Ergebnis zeigte sich, dass neben dem Faktor „Arbeitsmarkt" weitere Gründe, wie „Familie/Partnerschaft“ oder „Immobilie“, eine bedeutende Rolle für die Wanderungen aus der Untersuchungsgemeinde in ihre Nachbarkommunen spielten. So entfielen auf die beiden zuletzt genannten Kategorien zirka zwei Drittel aller Antworten. Bei einem durchgeführten Quervergleich der Motive „Familie/Partnerschaft“" und „Immobilie“ konnten zudem Zusammenhänge festgestellt werden. Beispielsweise zog ein Lebenspartner häufig in die Wohnung oder das Haus des

\footnotetext{
13 Die Höhe der Säulen stellt die Anzahl der aus der Stadt Wunsiedel in die jeweilige Rasterzelle Weggezogenen dar. Im Fallbeispiel sind die höchsten Säulen im Nahbereich um Wunsiedel zu erkennen.

14 Die Herkunftsadressen von zugezogenen Personen oder der Wohnort nach Wegzug werden im Melderegister geführt und wurden von der Stadtverwaltung Wunsiedel zur Verfügung gestellt.
}

Partners bzw. es wurde ein neues Haus am Wohnort des jeweiligen Partners errichtet (Koppers/Bergfeld/Höcht et al. 2014: $3 \mathrm{ff}$.).

Durch die Auswertung aller genannten Gründe konnten ,nicht adäquate“15 Immobilien und ein folglich nicht funktionierender Immobilienmarkt innerhalb der Stadt als prioritäres Handlungsfeld isoliert, weitere Untersuchungen hinsichtlich der Immobiliensituation in der Stadt durchgeführt und Maßnahmen zur Verbesserung eingeleitet werden. Eine dieser Maßnahme stellt die Gründung eines kommunalen Unternehmens zur Immobilienentwicklung in der Stadt Wunsiedel dar.

\subsection{Schulzuwegung}

Bezogen auf die Gemeinde Flossenbürg war in Kapitel 2 argumentiert worden, dass durch GIS-basierte Aufbereitung und Analyse von Meldedaten Erreichbarkeitsanalysen möglich werden, die sensitiv für Lebensphasen von Bewohnern in Einzugsgebieten sind. Als Beispiel diente die Berechnung der fußläufigen Erreichbarkeit einer Sportstätte. Nicht alle relevanten Infrastruktureinrichtungen werden jedoch primär zu Fuß aufgesucht. In diesen Fällen gilt es deshalb, auch Fahrtzeiten zu berücksichtigen, die bei der Nutzung des motorisierten Individualverkehrs (MIV) oder des

\footnotetext{
15 Als „nicht adäquat“ sind renovierungsbedürftige Wohnungen, deren Bestand in der Untersuchungsgemeinde relativ groß ist, anzusehen. Weiterhin äußerten die Befragten, dass keine ihren Anforderungen entsprechenden Wohnungsgrößen und Wohnungsgrundrisse auf dem Immobilienmarkt der Stadt vorhanden seien. Dies galt sowohl für Mietals auch für Kaufinteressenten.
} 
Abbildung 6 Fahrtroute zur am schnellsten erreichbaren Mittelschule - ausgehend von Wohnorten im Landkreis Tirschenreuth mit einer maximalen Pkw-Fahrtzeit von 15 Minuten Quelle: Höcht/Weichert/ Pickelmann (2012: 310)

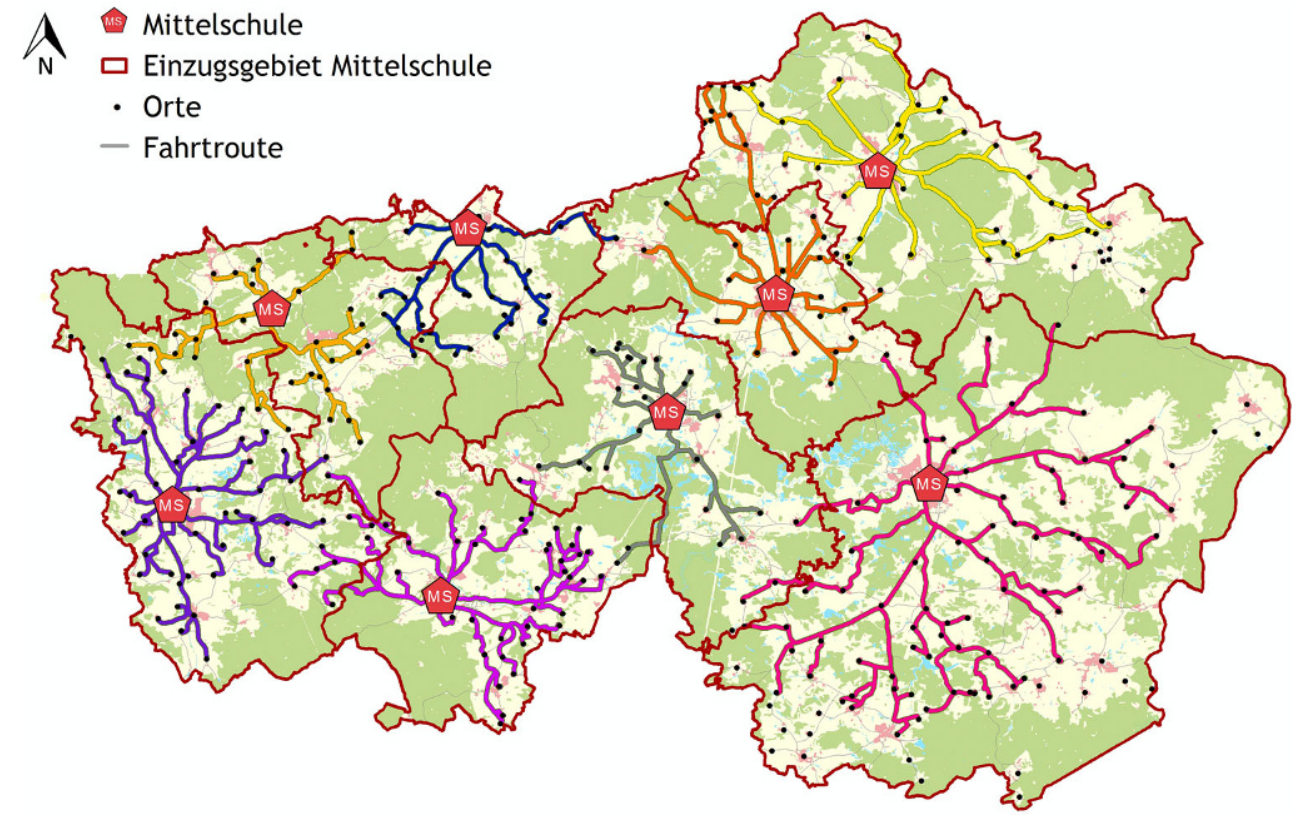

öffentlichen Personennahverkehrs (ÖPNV) verursacht werden. Die Fahrt vom Wohnort zu Infrastruktureinrichtungen, wie Sporthallen oder Schulen, erfolgt zudem oftmals über Gemeindegrenzen hinweg. Insbesondere bei der Schulplanung ist der Blick über die Gemeindegrenze unerlässlich, da manchen Schultypen übergemeindliche Einzugsgebiete, sogenannte Schulbezirke bzw. Schulsprengel, zugewiesen sind. ${ }^{16}$

Durch eine straßennetzbasierte Berechnung von Fahrtwegen zu Schulstandorten in einem Geographischen Informationssystem lassen sich Informationen generieren, die herkömmliche Ansätze der Schulwegeplanung sinnvoll ergänzen. ${ }^{17}$ So zeigt Abbildung 6 die Erreichbarkeit von Mittelschulen im nordbayerischen Landkreis Tirschenreuth, der aus 26 Gemeinden besteht - exemplarisch umgesetzt für den motorisierten Individualverkehr und einer im konkreten Fall als noch angemessenen definierten Fahrtzeit von bis zu 15 Minuten (vgl. Höcht/Weichert/Pickelmann 2012). Die gemeinsame Darstellung derzeitiger Schulsprengel („Einzugsgebiet Mittelschule“) mit den berechneten Fahrtzeiten ${ }^{18}$ offenbart, dass die Schulsprengel zwar

\footnotetext{
16 Schulsprengel: per Rechtsverordnung (Bayern: $§ 42$ BayEU - Bayerisches Gesetz über das Erziehungs- und Unterrichtswesen) räumlich abgegrenztes Gebiet für jede Grund- und Hauptschule (Mittelschule) sowie für Sonderschulen. In anderen Bundesländern gelten abweichende Bestimmungen.

17 So sind Erreichbarkeitsberechnungen auf dem Straßennetz für zahlreiche Anwendungsfälle neben der Schulwegeplanung mittlerweile etabliert (vgl. z. B. Schwarze 2008; Neumeier 2017).

${ }_{18}$ Die farblichen Unterscheidungen der Fahrtrouten dienen der differenzierten Darstellung der Zuwegung zu den einzelnen Destinationen, in diesem Falle Mittelschulen.
}

gemeindeübergreifend angelegt sind, sie aber nicht immer kürzeste Beförderungswege vorsehen. So sind in der Karte Wohnorte zu erkennen, die in benachbarten Schulsprengeln und nicht im Sprengel der zugeordneten Schule liegen. Schüler aus diesen Orten könnten folglich schneller zu einer anderen Schule mit dem Pkw gebracht werden, als dies derzeit vorgesehen ist.

Vergleichbare Analysen lassen sich auch für den ÖPNV durchführen. Sie müssen allerdings weitere Parameter - insbesondere Haltestellen, Fahrpläne sowie Warte- und Umsteigezeiten - berücksichtigen. Die Relevanz entsprechender Ansätze machen Projektarbeiten aus dem ländlichen Raum Sachsen-Anhalts deutlich, die zeigten, dass derzeitige Zuschnitte von Schulsprengeln eine ÖPNV-Beförderungszeit von zirka 2x60 Minuten (eine Stunde für den Hinweg und eine weitere Stunde für den Rückweg) zwischen manchen Haltestellen und Schulen zur Folge haben (Höcht/Weichert 2013).

Bei Verwendung von Meldedaten können Wohnadressen von Schülern identifiziert und deren Entfernung zu Haltestellen zusätzlich in die Berechnung einbezogen werden. In den Projektarbeiten im ländlichen Raum Sachsen-Anhalts wurden diese nicht berücksichtigt, weshalb die tatsächlich benötigten Reisezeiten zu den Schulen vermutlich sogar noch höher ausfallen dürften. Einwohnermeldedaten vermögen jedoch nicht nur, Reisezeitberechnungen (als Grundlage der Schulsprengelausweisung) zu ergänzen und Reisezeiten aus der Sicht der zu Befördernden wirklichkeitsnäher zu gestalten. Weiterführende Berechnungen von Schulwegen sind sogar nur auf diese Weise möglich. Dies gilt beispielsweise für zusätzliche Haltestellen an etablierten Bus-Beförderungsrouten, die dort zu verorten sind, wo 
sie von möglichst vielen Schülern schnell erreicht werden können. Dies gilt ferner, insofern optimierte Beförderungsrouten angeboten werden sollen, die unter Beachtung von Schülerwohnadressen berechnet werden. ${ }^{19}$

\subsection{Einwohnermeldedaten in einer Geodateninfrastruktur}

Die dargestellten Beispiele aus den Themenbereichen „Wanderungen“ und „Schulzuwegung“ visualisieren und quantifizieren demographische „Raumsituationen“ auf inner- und überkommunaler Ebene. Während für die Wanderungsuntersuchung nur das Melderegister einer einzelnen Kommune als Basis der Auswertung diente, bedarf es für die melderegisterbasierte Optimierung von übergemeindlichen Schulsprengeln der Meldedaten mehrerer Kommunen. Der dabei für die Datenbereitstellung zu betreibende Aufwand kann beträchtlich sein. So begründen in der Praxis abweichende Melderegistersoftware und unterschiedliche Anwenderfertigkeiten in den Einwohnermeldeämtern mitunter lange Datenbeschaffungszeiträume, die iterierenden Analyseprozessen und dem Monitoring demographischer Entwicklungen auf der Basis von Meldedaten entgegenstehen. Dass diesen Herausforderungen jedoch erfolgreich begegnet werden kann, zeigt die prototypische Bereitstellung demographischer Fachdaten als Teil der Geodateninfrastruktur (GDI) des Landes Sachsen-Anhalt (vgl. Vilser 2014).

Eine Geodateninfrastruktur ist ,eine aus technischen, organisatorischen und rechtlichen Regelungen bestehende Bündelung von Geoinformationsressourcen, in der Anbieter von Geodatendiensten mit Nachfragern solcher Dienste kooperieren“.20 Zentrale Geoinformationsressource sind im konkreten Fall geokodierte Meldedaten. Die Datenübertragung als entscheidender Aspekt der Kooperation zwischen Anbietern (den Kommunen) und Nutzern (die Landesplanung) erfolgt über das Internet. In einer Studie (Wegmann 2017) wurde die technische Machbarkeit der automatisierten und landesweiten Verfügbarmachung kom-

\footnotetext{
19 Ohne kleinräumige Bevölkerungsdaten erscheint es zudem fraglich, wie die derzeit diskutierten Vorschläge für eine bessere ambulante medizinische Versorgung effizient umzusetzen sind. So sollen $99 \%$ der Bevölkerung Deutschlands Praxen von Kinder- und Frauenärzten künftig innerhalb von nur 20 Minuten erreichen können (Mihm 2018). Ohne das Wissen darüber, wo Kinder und Frauen (gegebenenfalls in besonders nachfragenden Altersgruppen) wohnen, dürften sich minutengenaue Erreichbarkeiten für die Gesamtbevölkerung, jedoch nicht passgenau für die relevante Zielgruppe berechnen lassen. Infolgedessen müssten die relevanten Facharztpraxen flächendeckend vorgehalten werden - beispielsweise selbst im Einzugsgebiet von alternden Einfamilienhausgebieten, in denen womöglich (derzeit) keine Kinder mehr wohnen.

$20 \mathrm{Vgl}$. http://www.geoinformatik.uni-rostock.de/einzel.asp?ID=441 (29.06.2018).
}

munaler Einwohnermeldeinformationen ausgehend von den Projektkommunen Gardelegen und Möckern geprüft. Die Umsetzung erfolgte durch ein Vorgehen in drei Schritten:

Zunächst wurden Meldedaten durch eine neu entwickelte und in den Kommunen installierte Software, die die dort etablierte Einwohnermeldesoftware ergänzte, automatisiert aufbereitet. Dieser Schritt umfasste sowohl die Geokodierung ${ }^{21}$ der Datenbasis, die Aggregation auf vorgegebene Raumeinheiten als auch die Umsetzung datenschutzrechtlicher Vorgaben. Die datenschutzkonforme Bereitstellung erfolgte durch technisches Umsetzen der im Projekt durch den verantwortlichen Datenschutzbeauftragten definierten (aber vorläufigen) Vorgaben. Ziel war der Nachweis der technischen Machbarkeit einer nahtlosen Umsetzung entsprechender Vorgaben. Eine wesentliche Voraussetzung ist die Aufbereitung der Daten noch in der melderegisterführenden Stelle und vor dem Übertragen der Daten an Dritte. Die Detailvorgaben bezüglich des Datenschutzes können sich jedoch nach Abschluss des Projekts ändern und weiterentwickeln, weshalb an dieser Stelle nicht weiter auf sie eingegangen wird. ${ }^{22}$

Dieser erste Schritt führt zum Überwinden des eingangs erwähnten Aufbereitungsaufwandes, der den Praxiseinsatz von Einwohnermeldedaten in der raumbezogenen Planung ländlicher Kommunen bislang hemmt. Anschließend werden die demographischen Daten mittels raumbezogener Darstellungs- und Download-Dienste ${ }^{23}$ über das Internet verwaltungs- und GIS-Software-übergreifend bereitgestellt (Schritt 2), wodurch sie in einem dritten Schritt in einem Web-Viewer betrachtet oder in Desktop-GIS-Software der Landesplanung (prinzipiell aber auch anderer Verwaltungseinheiten, Kommunen und sonstiger Nutzungsbefugter) eingeladen und dort analytisch weiterbearbeitet werden können.

Darüber hinaus wurde eine Zwischenlösung geschaffen, die die Einfachheit des Web-Viewers mit relevanten, aber intuitiv zu bedienenden Funktionen eines DesktopGIS kombiniert. Diese umfassen ein Spektrum an zentralen Funktionalitäten, die gemeinsam mit potenziellen Nutzern definiert wurden (vgl. Kirschke 2015). So erlaubt der entwickelte Web-Client (vgl. Abbildung 7), dass weitere

\footnotetext{
${ }^{21}$ Hierfür wurde der Geokodier-Service des Landes Sachsen-Anhalt, eine weitere Komponente der Landes-Geodateninfrastruktur, verwendet (vgl. Kügeler/Roth/Voigt 2015).

${ }^{22}$ Beispielsweise sollte bei einer zu geringen Anzahl von Bewohnern des gleichen Geschlechts und der gleichen Altersklasse keine entsprechende demographische Information für den betroffenen Ortsteil bereitgestellt werden.

${ }^{23}$ Diese wurden als sogenannte „Web Map“ und „Web Feature Services" des Standardisierungsgremiums OGC (Open Geospatial Consortium) bzw. als View- und Download-Services der europäischen Initiative INSPIRE (Infrastructure for Spatial Information in the European Community) implementiert.
} 


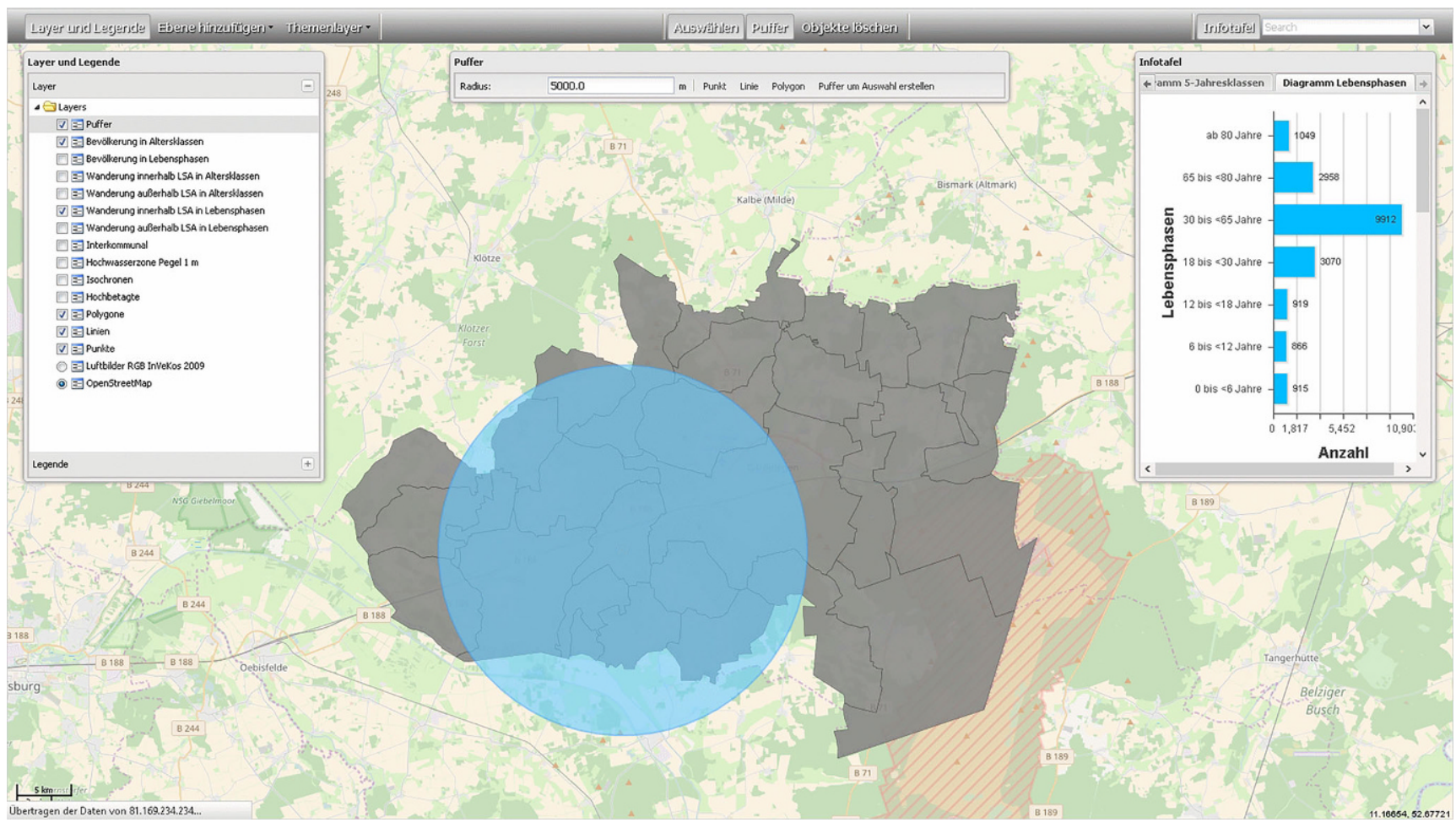

Abbildung 7 Demographieorientiertes Web-GIS auf der Basis kommunaler Melderegister (Prototyp). Selektion der Bevölkerungszahl nach Lebensphasen: hier in den durch Puffer berührten Ortsteilen von Gardelegen im Land Sachsen-Anhalt

Geodatendienste der Landesverwaltung in die Anwendung integriert und gemeinsam mit den demographischen Daten genutzt werden. Zudem lässt sich die Anzahl von Personen in bestimmten Altersklassen und Lebensphasen für die Ortsteile der Modellkommunen Gardelegen (vgl. Abbildung 7) und Möckern abfragen. Mittels Pufferbildungen können die Raumeinheiten, auf die sich die statistischen Informationen beziehen, zudem flexibel bestimmt werden.

\section{Umgang mit der künftigen Bevölkerungsentwicklung im ländlichen Raum: Grenzen kommunaler Bevölkerungsstudien?}

Die zuvor dargestellten Untersuchungen beruhen auf der Betrachtung gegenwärtiger Bevölkerungssituationen. Ihre Diskussion mit Praktikern aus Planung und Kommunalpolitik ruft jedoch nicht selten den Wunsch nach entsprechenden Analysen auch für die Zukunft hervor (Schaffert/Müller/Benndorf 2011). Kleinräumige Bevölkerungsvorausberechnungen sind jedoch mit Unsicherheiten verbunden, die aus der häufig kleinen Grundgesamtheit und der Bedeutung von Wanderungsbewegungen für die gemeindliche Bevölkerungsentwicklung resultieren. Schließt ein kommunal bedeutender Arbeitgeber seine Niederlassung, kann dies Bevölkerungsentwicklungen beeinflussen
(Jain/Schmithals 2009: 315). In kleineren Gemeinden gilt dies auch für den Bau von Pflegeeinrichtungen, wie nachfolgend am Beispiel der Marktgemeinde Teisnach im ländlichen Niederbayern skizziert wird.

Die künftige Bevölkerungsstruktur der Gemeinde wurde ausgehend vom Jahre 2012 - damals hatte die Gemeinde knapp 2.900 Einwohner - mit einem Programm berechnet, das das Melderegister als Datengrundlage nutzt und auf der verbreiteten Kohorten-Komponenten-Methode basiert. ${ }^{24}$ Die Vorausberechnung erfolgte für alle Gemeinden des zugehörigen Landkreises Regen, wobei als Annahme für künftige Wanderungszahlen jeweils Mittelwerte der jährlichen Wanderung der vorausgegangenen zehn Jahre Eingang in die Berechnung fanden.

Während diese Vorgehensweise für die restlichen $23 \mathrm{Ge}-$ meinden des Landkreises zu plausiblen Ergebnissen führte, überraschte Teisnach mit einer auffällig hohen Zahl an hochbetagten Frauen im Jahr 2035 (vgl. Abbildung 8a-

\footnotetext{
${ }^{24}$ Danach berechnet sich die künftige Bevölkerungsstruktur, indem von einer Ausgangsbevölkerung ausgegangen wird und die Komponenten „Sterbefälle“, „Geburten“, „Zuzüge“ und „Wegzüge“ berücksichtigt werden. Die Komponenten werden für jedes Jahr getrennt berechnet, bevor sie zum nächsten Zeitschnitt in die Vorausschätzung der Bevölkerung eingehen. Zudem werden Annahmen zu künftigen Veränderungen der Komponenten getroffen (Flöthmann/Tovote/ Schleifnecker 2006: $21 \mathrm{ff}$.). Konzeption und Funktionsweise des Programms sind in Benndorf/Bergfeld/Schaffert et al. (2013) beschrieben.
} 

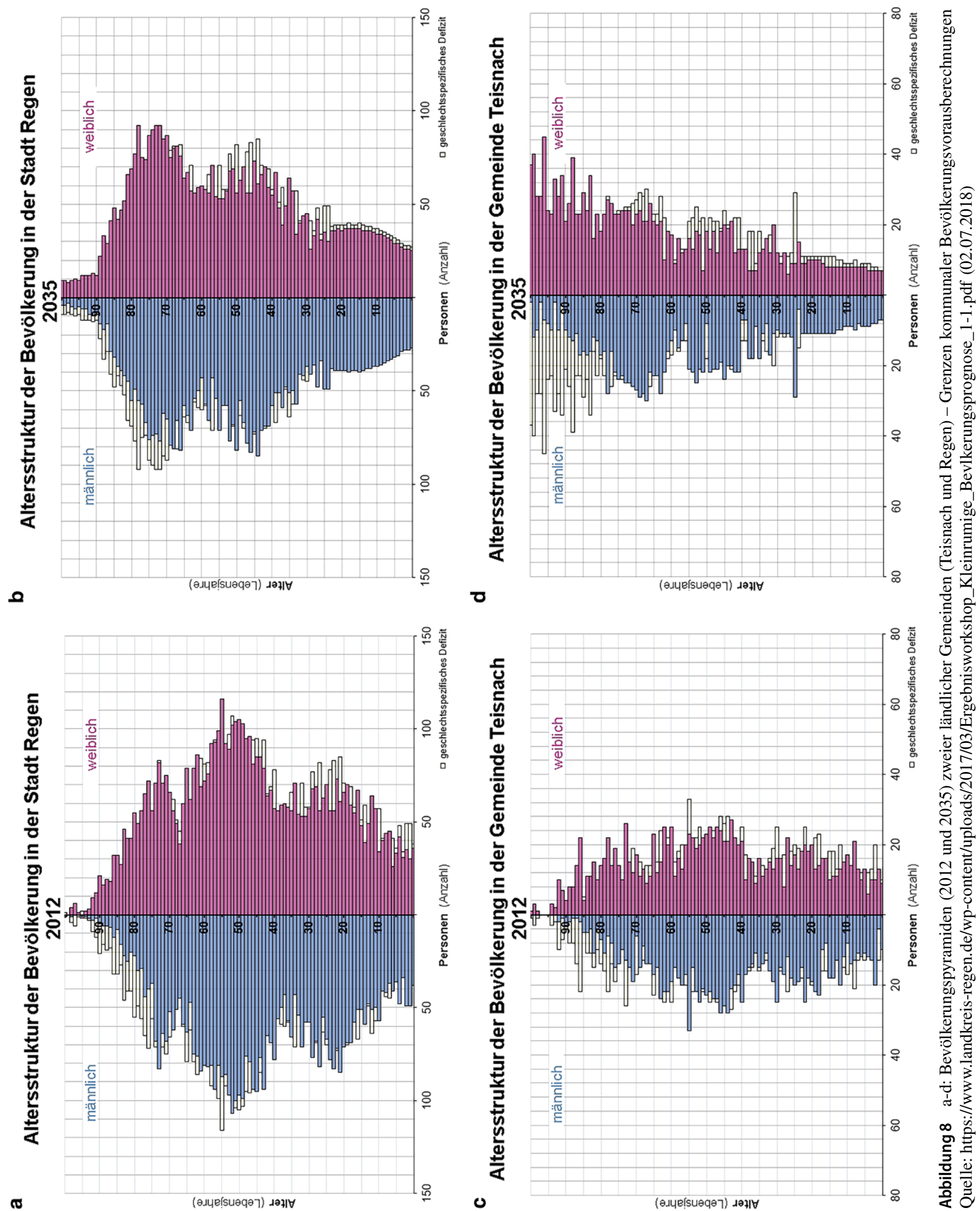

d). Diese Abbildung zeigt Alterspyramiden der Untersuchungsgemeinde für das Jahr 2012 und 2035; zudem werden zur Einordnung Alterspyramiden auch der zugehörigen Kreisstadt Regen (rund 10.900 Einwohner) dargestellt.

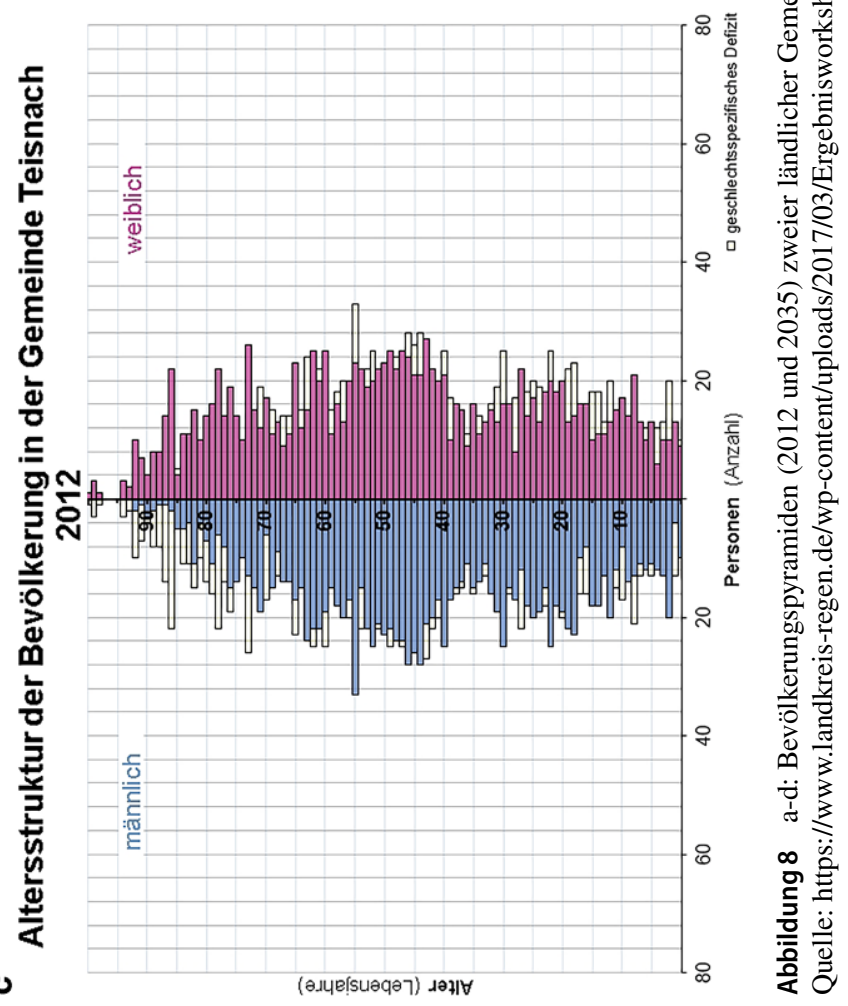

Das Resultat ist im zuvor erfolgten, überproportional starken Zuzug durch hochbetagte Frauen begründet. So stellte diese Bevölkerungsgruppe - bei insgesamt niedrigeren Zuwanderungszahlen - einen hohen Anteil am Zuzug in besagte Gemeinde in dieser Zeit. Die adressgenaue Infor- 
mationsquelle Melderegister half zudem, dieses Phänomen zu lokalisieren und fachlich einzuordnen. So erfolgten Zuzüge dieser Art an nur drei Adressen im Ort, allesamt Standorte von Pflegeheimen. Während es durchaus möglich erscheint, dass die Pflegeheime die Bevölkerungsstruktur der Gemeinde dauerhaft signifikant verändern, dürfte der erfolgte Zuzug von deutlich mehr Frauen als Männer eher ein zeitlicher Zufall oder in zielgruppengerechten Angeboten des Heims begründet sein, die sich jederzeit ändern können.

Solche Unsicherheiten machen kleinräumige demographische Vorausberechnungen anfällig, aber nicht obsolet. Schließlich dürfte der Bedarf in vielen Kommunen, die künftige Auslastungen von Infrastrukturen abschätzen möchten, gegeben sein. Dieser Bedarf, so die Erfahrungen aus dem Landkreis Regen, bezieht sich dabei auch auf innerörtliche Gebiete und deren Zusammenschau mit Ergebnissen der gesamten Gemeinde. Deshalb wurde das Melderegister auch hier anfangs geokodiert, wodurch sich die Bewohner der einzelnen Ortsteile mittels räumlicher Abfrage in einem Geographischen Informationssystem effizient ermitteln ließen. ${ }^{25}$ Zudem deckten Kartendarstellungen die Möglichkeit von differenzierten zeitlichräumlichen Entwicklungspfaden (hier: wachsende Ortsteile in demographisch schrumpfenden Kommunen) und deren räumliche Muster auf.

Für die seriöse Verwendung von Vorausberechnungen für solche kleinräumigen Gebiete ist aber die Einsicht notwendig, dass ihre Resultate keine singulären Wahrheiten darstellen, sondern nur als Orientierungshilfen zu verstehen sind. Das Anbieten von mehr als nur einer Vorausberechnung, wie sie sich bereits durch unterschiedliche Wanderungsannahmen in der genannten Software realisieren lässt - und beispielsweise in der 13. koordinierten Bevölkerungsvorausberechnung des Statistischen Bundesamts durch Varianten umgesetzt wird (Statistisches Bundesamt 2015) ist dabei aber nur als erster Schritt zu sehen. Vielmehr erscheint ein solides Verständnis derjenigen Faktoren, die die demographische Entwicklung einer Gemeinde beeinflussen, notwendig, um flexibel auf sich verändernde Rahmenbedingungen zu reagieren. Ebenso müssen derzeit noch nicht naheliegende, aber im Realisierungsfalle durchschlagsstarke Entwicklungen (wie marktreife altersgerechte Assistenzsysteme) und Ereignisse (z. B. den politischen Beschluss zu Ausbau und Förderung dieser in der Fläche) mit bedacht und ihre Konsequenzen für die demographische Situation vor Ort abgeleitet werden. Einen solchen Ansatz verfolgt

\footnotetext{
25 Anhand von Umringen der Ortsteile, die aus dem Projekt OpenStreetMap stammen, wurde die Zugehörigkeit der Adresspunkte zu diesen innerörtlichen Gebieten in einem Geographischen Informationssystem räumlich abgefragt. Dies erfolgte, da ein Attributfeld „Ortsteil" in den Meldedaten zwar angelegt, im konkreten Fall aber nicht immer mit Werten belegt war.
}

die informelle Planungsmethode der Szenariotechnik, die mittlerweile auf eine jahrzehntelange Entwicklungs- und Erfolgsgeschichte in der Unternehmensplanung, in Klimaund Umweltwissenschaften zurückblicken kann. Ihre unterschiedlichen Einsatzgebiete machen jedoch differenzierte Ausgestaltungen ihres methodischen Grundkorsetts notwendig. In den genannten Erfolgsfeldern sind solche fachspezifischen Adaptionen erfolgt. In der Raumplanung wird sie jedoch noch immer meist fachunspezifisch, im Stile eines „Swiss pocket knife“ (van Notten 2006: 84) eingesetzt, was einen Grund für die bislang nur partiellen Erfolge der Methode in raumplanerischen Anwendungen darstellen dürfte. Der Vorschlag von Schaffert (2011a, 2015) blickt auf Wohngebäude im demographisch schrumpfenden ländlichen Raum und passt den Methodenrahmen für diesen konkreten Anwendungsfall und die Zielgruppe der politischen Entscheider an. Andere Akteurkonstellationen und abweichende Fragestellungen machen jedoch jeweils spezifische Ausgestaltungen notwendig. In diesem Bereich besteht deshalb nach wie vor Forschungsbedarf.

\section{Fazit und Ausblick}

Die Verwendung geokodierter Melderegisterdaten trägt dazu bei, eine zentrale Informationslücke in der Planungsrealität ländlicher Gemeinden zu schließen, da sie - anders als demographische Daten der Statistikämter von Bund und Ländern - Untersuchungen auch innerörtlicher demographischer Gegebenheiten erlauben. Je nach Fragestellung, Planungszweck und Datenschutzvorgaben lassen sich Einwohnermeldedaten ausgehend von der Adresse flexibel auf höhere Raumeinheiten aggregieren. Diese Flexibilität ist nach der Adress-Geokodierung der Melderegister technisch durch die Funktionalitäten etablierter GIS-Programme gegeben. Allerdings hängt sie auch von der Verfügbarkeit von Geodaten ab, die solche höheren Raumeinheiten repräsentieren. Durch Entwicklungen in den Bereichen Open Government Data, Crowd Sourced bzw. Volunteered Geographic Information und die INSPIRE-Initiative sind heute eine Vielzahl solcher Geodaten - z. B. die Verwaltungsgebiete 1:250.000 des Bundesamtes für Kartographie und Geodäsie oder Bebauungsplanumringe, die durch eine steigende Zahl von Gemeinden in überkommunale Geodateninfrastrukturen eingebracht werden - über das Internet und teilweise kostenfrei erhältlich.

Durch konsequentes Befolgen von technischen Standards im Rahmen von Geodateninfrastrukturen können Einwohnermeldedaten auch gemeindeübergreifend bereitgestellt und von berechtigten Nutzern verwendet werden. Auf diese Weise lassen sich Planungsinformationen generieren, die eine horizontal und vertikal flexible Raumplanung unterstützen. „Horizontal“ beschreibt in diesem 
Zusammenhang die Synergien, die sich durch das grenzübergreifende Planen auf derselben administrativen Ebene, namentlich dem interkommunalen Zusammenarbeiten, ergeben. „Vertikal“ steht für die Möglichkeit, dabei sowohl die örtliche als auch regionale Planungsebene mit demographischen Daten in gewünschter räumlicher Informationstiefe zu bedienen.

\section{Literatur}

Bauer, U.; Holz-Rau, C.; Scheiner, J. (2005): Standortpräferenzen, intraregionale Wanderungen und Verkehrsverhalten. In: Raumforschung und Raumordnung 63, 4, 266-278. doi: 10.1007/BF03183 813

Benndorf, F.; Bergfeld, A.; Schaffert, M.; Koppers, L. (2013): Schlussbericht des BMBF-Forschungsprojekts „DemoGI - Geoinformatik-Methoden und -Werkzeuge zur Analyse, Anpassung und Bewältigung des demographischen Wandels". Dessau-Roßlau.

Benneter, S. (2013): Planung auf den Punkt gebracht - mit verorteten Einwohnermeldedaten zu passgenauen Planungsgrundlagen. In: Meinel, G.; Schumacher, U.; Behnisch, M. (Hrsg.): Flächennutzungsmonitoring V: Methodik - Analyseergebnisse - Flächenmanagement. Berlin, 245-251. = IÖR-Schriften 61.

Bürkner, H.-J.; Berger, O.; Luchmann, C.; Tenz, E. (2007): Der demographische Wandel und seine Konsequenzen für Wohnungsnachfrage, Städtebau und Flächennutzung. Erkner. https://leibniz-irs. de/fileadmin/user_upload/IRS_Working_Paper/wp_wandel.pdf (28.06.2018).

Burgdorf, A. (2010): Disaggregation von Bevölkerungsdaten mittels ATKIS Basis DLM. In: Strobl, J.; Blaschke, T.; Griesebner, G.; Zagel, B. (Hrsg.): Angewandte Geoinformatik 2010. Berlin/ Offenbach, 474-483.

Farrokhikhiavi, R.; Dietrich, C.; Vallée, D. (2013): Neue Ansätze zur Sicherung der Daseinsvorsorge. In: Flächenmanagement und Bodenordnung 75, 5, 193-200.

Flöthmann, E.-J.; Tovote, U.; Schleifnecker, T. (2006): Ein Blick in die Zukunft. Deutschlands Kommunen im Wettbewerb um Einwohner. Ergebnisse der kleinräumigen Bevölkerungsprognose 2020. In: Bertelsmann-Stiftung (Hrsg.): Wegweiser Demographischer Wandel 2020. Gütersloh, 13-23.

Föbker, S. (2008): Wanderungsdynamik in einer schrumpfenden Stadt - eine Analyse innerstädtischer Umzüge. Dissertation, Rheinische Friedrich-Wilhelms-Universität Bonn.

Haußmann, M. (2012): Die Städtestatistik als Datennutzer und Informationsprovider - Wie können die bestehenden Potenziale genutzt werden? In: Rat für Sozial- und Wirtschaftsdaten (Hrsg.): Georeferenzierung von Daten. Situation und Zukunft der Geodatenlandschaft in Deutschland. Berlin, 74-79.

Höcht, V. (2016a): Strategisches Demographie-Management für kleine Kommunen in schrumpfenden Regionen. Ein interdisziplinärer Ansatz als Beitrag für eine Demographie orientierte Stadtentwicklungsplanung. Darmstadt. = Schriftenreihe der Fachrichtung Geodäsie, Fachbereich Bauingenieurwesen und Geodäsie, Technische Universität Darmstadt 50.

Höcht, V. (2016b): Die bevölkerungsbezogene, strategische Steuerung der Stadtentwicklung in kleinen Kommunen in Schrumpfungsregionen auf Basis des kommunalen Melderegisters. In: Flächenmanagement und Bodenordnung 78, 3, 111-118.

Höcht, V.; Kirschke, T. (2017): Flexible Geoinformationssysteme in der kommunalen Planung. In: VDV-Magazin 67, 2, 116-122.

Höcht, V.; Weichert, T. (2013): 57 Minuten mit dem Bus zur Schule? Das ist zu lang! Die Anpassung von Infrastrukturen in ländlichen Räumen: GIS-Werkzeuge für die Schulstandortplanung. In: Pasternack, P.; Maue, I. (Hrsg.): Lebensqualität entwickeln in schrumpfenden Regionen. Wittenberg, 48-53. = Schriftenreihe des WZW 11.

Höcht, V.; Weichert, T.; Pickelmann, U. (2012): Wie weit ist es bis zur Schule? GIS-basierte Analysen der Schulzuwegung mit Pkw und ÖPNV. In: VDV-Magazin 63, 4, 308-311.

Huber, S. (2015): Entwicklung einer zentralen Adressdatenverwaltung für kommunale Planungsaufgaben. Praktische Projektarbeit, Technische Universität München. https://mediatum.ub.tum.de/ doc/1291311/1291311.pdf (28.06.2018).

Jain, A.; Schmithals, J. (2009): Motive für die Wanderung von Westnach Ostdeutschland und Rückkehrtypen. In: Cassens, I.; Luy, M.; Scholz, R. (Hrsg.): Die Bevölkerung in Ost- und Westdeutschland. Wiesbaden, 313-333. doi: 10.1007/978-3-531-91832-7_13

Karrais, N. (2014): Modellierung der verbrauchsbasierten Erreichbarkeit mit GIS. In: Strobl, J.; Blaschke, T.; Griesebner, G.; Zagel, B. (Hrsg.): Angewandte Geoinformatik 2014. Berlin/Offenbach, 5667.

Kirschke, T. (2015): Konzeption und Entwicklung eines webbasierten Unterstützungsinstruments für Fachanwender von Bevölkerungsdaten. In: Zeitschrift für Geodäsie, Geoinformation und Landmanagement 140, 5, 297-305.

Kleinwächter, D. (2014): Baulücken- und Leerstandskataster - Dienstangebote für niedersächsische Kommunen. In: Meinel, G.; Schumacher, U.; Behnisch, M. (Hrsg.): Flächennutzungsmonitoring VI. Innenentwicklung - Prognose - Datenschutz. Berlin, 85-92. $=$ IÖR-Schriften 65 .

Köhler, T. (2014): Geoinformation in der kommunalen Abfallentsorgungs- und strategischen räumlichen Planung. In: Raumforschung und Raumordnung 72, 6, 491-501. doi: 10.1007/s13147014-0320-9

Köhler, T.; Schaffert, M. (2015): Building Measures in the Face of Population Decline. In: Hepperle, E.; Dixon-Gough, R.; Mansberger, R.; Paulsson, J.; Reuter, F.; Yilmaz, M. (Hrsg.): Challenges for Governance Structures in Urban and Regional Development. Zürich, 209-222.

Kötter, T.; Schollän, U. (2010): Suburbane Wohngebiete in Zeiten des demographischen Wandels - Szenarien, Handlungsbedarfe und Anpassungsstrategien. In: Flächenmanagement und Bodenordnung 72, 6, 254-260.

Koppers, L.; Baumann, H. (2011): GIS und demographischer Wandel. In: Zeitschrift für Geodäsie, Geoinformation und Landmanagement 136, 4, 218-223.

Koppers, L.; Bergfeld, A.; Höcht, V.; Benndorf, F. (2014): Wanderungsmotivuntersuchung Wunsiedel. https://www.stmi.bayern.de/ assets/stmi/buw/staedtebau/broschuere_wunsiedel.pdf (14.08.2017).

Kügeler, A.; Roth, M.; Voigt, J. (2015): Zentraler Geodatenknoten Sachsen-Anhalt. Fachliches Feinkonzept. https://www.gfds. sachsen-anhalt.de/ows/de/datei/download/id/3939,501/zentraler_ geodatenknoten_lsa_feinkonzept_v_1.0.pdf (29.06.2018).

Lang, M.; Zettler, L. (2012): Dorferneuerung Niederraunau. Ein Modellprojekt zur Innenentwicklung am Beispiel „Untere Gasse“. In: Mitteilungen des DVW Bayern 64, 4, 335-346.

Mihm, A. (2018): Umbau im Gesundheitswesen: Jeder soll einen Hausarzt in 15 Minuten erreichen. In: Frankfurter Allgemeine Zeitung vom 4. Mai 2018. http://www.faz.net/aktuell/wirtschaft/ hausaerzte-sollen-bald-hoechstens-15-minuten-fuer-jeden-entferntsein-15568844.html (02.07.2018).

Neufeld, M.; Beyrich, L.; Burkhardt, N.; Engl, C.; Gramann, P.; Chilla, T. (2015): Leerstandmanagement im Ländlichen Raum. GISgestützte Anpassungsmaßnahmen im Demographischen Wandel. In: Fränkische Geographische Gesellschaft (Hrsg.): Mitteilungen der Fränkischen Geographischen Gesellschaft 60. Erlangen, 91100.

Neumeier, S. (2017): Regionale Erreichbarkeit von ausgewählten Fachärzten, Apotheken, ambulanten Pflegediensten und weiteren ausgewählten Medizindienstleistungen in Deutschland: Ab- 
schätzung auf Basis des Thünen-Erreichbarkeitsmodells. Braunschweig. $=$ Thünen Working Paper 77 .

van Notten, P. (2006): Scenario development: a typology of approaches. In: OECD - Organisation for Economic Co-Operation and Development (Hrsg.): Schooling for tomorrow. Think scenarios, rethink education. Paris, 69-92. doi: 10.1787/9789264023642-en

o. V. (2018): Städte- und Gemeindebund rät: Kommunen sollen Daten der Bürger verkaufen. In: Frankfurter Allgemeine Zeitung vom 9. April 2018. http://www.faz.net/aktuell/wirtschaft/diginomics/ kommunen-sollen-daten-der-buerger-verkaufen-15533156.html (02.07.2018).

Planinsek, S. (2011): Die Entwicklung von Eigenheimgebieten der 1960er- bis 1980er-Jahre in Gemeinden des Umlandes und der Peripherie: Generierung und Analyse von Bevölkerungs- und Siedlungsstrukturdaten auf Quartiersebene durch Geocoding. Fallstudien aus Baden-Württemberg. Dissertation, Karlsruher Institut für Technologie.

Raab, A. (2006): Nachfrageorientierte Innenentwicklung und Flächenmanagement. Zukunftsaufgabe bei Stadt- und Dorfumbau. In: Mitteilungen des DVW Bayern 65, 4, 547-563.

Rößler, C. (2011): Aktuelle Wanderungsmuster und Wanderungsmotive im Ländlichen Raum Sachsens. In: BBSR - Bundesinstitut für Bau-, Stadt- und Raumforschung (Hrsg.): Demografische Spuren des ostdeutschen Transformationsprozesses. 20 Jahre Deutsche Einheit. Bonn, 66-74.

Rudolph-Cleff, A. (2012): Ortsbestimmung. Zum Einsatz von Geoinformationssystemen in der Stadtplanung. In: Flächenmanagement und Bodenordnung 74, 5, 193-199.

Schaffert, M. (2008): Auswirkungen von Bevölkerungsrückgang auf die technische Infrastruktur deutscher Kommunen. Vorarbeiten zur Entwicklung eines GIS-basierten Planungsinstruments zur Erstellung von Leerstandsszenarien. In: Allgemeine VermessungsNachrichten 11-12, 388-393.

Schaffert, M. (2010): Strategisches Leerstandsmanagement auf kommunaler Ebene. Komponenten, Anforderungen und Mehrwerte durch den Einsatz von Geoinformationssystemen. In: Bundesamt für Kartographie und Geodäsie (Hrsg.): Arbeitsgruppe Automation in Kartographie, Photogrammetrie und GIS - Tagung 2009. Frankfurt am Main, 108-117. = Mitteilungen des Bundesamtes für Kartographie und Geodäsie 45.

Schaffert, M. (2011a): Szenariotechnik und GIS - Ein Beitrag zur demographierobusten Planung in Kommunen. Darmstadt. = Schriftenreihe der Fachrichtung Geodäsie, Fachbereich Bauingenieurwesen und Geodäsie, Technische Universität Darmstadt 32.

Schaffert, M. (2011b): GIS-basiertes Leerstandsmanagement für ländliche Wohngebäude. Szenarien im Spannungsfeld öffentlicher und privater Interessen. In: Hepperle, E.; Dixon-Gough, R.; Kalbro, T.; Mansberger, R.; Meyer-Cech, K. (Hrsg.): Core-Themes of Land Use Politics: Sustainability and Balance of Interests. Zürich, 349-362.

Schaffert, M. (2015): Scenario Planning as a Tool for Handling Demographic Change in Rural German Municipalities. Advantages, Disadvantages and Increasing the Potential by Geo-information. In: Hepperle, E.; Dixon-Gough, R.; Mansberger, R.; Paulsson, J.; Reuter, F.; Yilmaz, M. (Hrsg.): Challenges for Governance Structures in Urban and Regional Development. Zürich, 195-208.

Schaffert, M.; Müller, M.; Benndorf, F. (2011): Kommunen im Zeichen des demografischen Wandels. Planungsunterstützung durch kombinierte Nutzung von Melderegister und Geobasisdaten. In: Strobl, J.; Blaschke, T.; Griesebner, G. (Hrsg.): Angewandte Geoinformatik 2011. Berlin/Offenbach, 503-512.

Scholz, R.; Kreyenfeld, M. (2016): Der registergestützte Zensus in Deutschland: Historische Einordnung und Bedeutung für die Bevölkerungswissenschaft. In: Comparative Population Studies 41, 3-34. doi: 10.12765/CPoS-2016-08de

Schwarze, B. (2008): Kleinräumige Erreichbarkeitsanalysen mit GIS. http://www.sv.bgu.tum.de/fileadmin/w00bnl/www/pictures/ Veranstaltungen/Paper_2.pdf (02.07.2018).

Spehl, H. (Hrsg.) (2011): Leerstand von Wohngebäuden in ländlichen Räumen. Beispiele ausgewählter Gemeinden der Länder Hessen, Rheinland-Pfalz und Saarland. Hannover. = E-Paper der ARL 12. Statistisches Bundesamt (2015): Bevölkerung Deutschlands bis 2060. 13. koordinierte Bevölkerungsvorausberechnung. Wiesbaden.

Sturm, G.; Meyer, K. (2009): Was können die Melderegister deutscher Großstädte zur Analyse residenzieller Multilokalität beitragen? In: Informationen zur Raumentwicklung 1/2, 15-29.

Vilser, I. (2014): Bereitstellung ortsteilbezogener Bevölkerungszahlen für die Landes- und Regionalplanung. In: Zeitschrift für das Öffentliche Vermessungswesen des Landes Sachsen-Anhalt 20, 2, 119-130.

Waldner, U. J.; Simonet, S.; Machguth, H.; Axhausen, K. W. (2005): Geokodierung von Adressen: Methodik und Erfolgsquoten bei unterschiedlichsten Datenquellen. Zürich. $=$ Arbeitsbericht Netzwerk Stadt und Landschaft 3. doi: 10.3929/ethz-a-005226679

Wegmann, C. (2017): Bereitstellung ortsteilbezogener Bevölkerungsdaten für die Landes- und Regionalplanung. In: Flächenmanagement und Bodenordnung 79, 5, 18-21.

Wehrhahn, R.; Sandner Le Gall, V. (2011): Bevölkerungsgeographie. Darmstadt.

Zakrzewski, P.; Berndgen-Kaiser, A.; Fox-Kämper, R.; Siedentop, S. (2014): Prospects for West German Post-War Single-Family Home Neighbourhoods. Revitalising Housing Stocks as a New Policy Field for Suburban and Rural Municipalities. In: Comparative Population Studies 39, 2, 285-318. doi: 10.12765/CPoS2014-06de 